\title{
The Alcohol Use Disorders Identification Test was not affected by ethnic and sex bias
}

\author{
Steinbauer JR, Cantor SB, Holzer CE III, et al. Ethnic and sex bias in primary care screening tests for alcohol use disorders. Ann \\ Intern Med 1998 Sep 1;129:353-62.
}

\section{Question}

Are there ethnic and sex biases in 3 self report screening tests for alcohol use disorders in a primary care population?

\section{Design}

Blinded comparison of the results of 3 self report screening tests with the diagnostic standard (diagnosis according to DSM-IV criteria).

\section{Setting}

University based family practice centre in Galveston, Texas, USA.

\section{Patients}

1333 adults (mean age 43 y) randomly selected from appointment lists stratified by sex and ethnicity (white, AfricanAmerican, and Mexican-American).

\section{Description of tests and diagnostic standard}

The 3 screening tests used were the CAGE questionnaire, the Self-Administered Alcoholism Screening Test (SAAST), and the Alcohol Use Disorders Identification Test (AUDIT). The diagnostic standard, an interview using DSM-IV criteria, was also administered by a project interviewer and was scored by computer algorithm.

\section{Main outcome measures}

Area under the receiver operating characteristic (ROC) curves, and positive and negative likelihood ratios.

\section{Main results}

The areas under the ROC curves for the CAGE questionnaire and the SAAST ranged from 0.61 to 0.88 and were poor for African-American men (0.69 and 0.68 , respectively) and Mexican-American women (0.68 and 0.61 , respectively). The area under the ROC curve for the AUDIT was $>0.9$ for each of the patient subgroups. Positive likelihood ratios (LRs) for the AUDIT (cutoff point 5) were $\geqslant$ those for the other screening tests (cutoff points 2 for the CAGE and 3 for the SAAST), whereas negative LRs were lowest for the AUDIT $(<0.33)$, indicating its superiority in ruling out a disorder (table).

\section{Conclusion}

The Alcohol Use Disorders Identification Test was not affected by ethnic and sex bias when screening for alcohol use disorders in primary care.

Sources of funding: In part, National Institute on Alcohol Abuse and Alcoholism and Bureau of Health Professions, Health Resources and Services Administration.

For correspondence: Dr RJ Volk, Department of Family Medicine and Community Medicine, Baylor College of Medicine, 5510 Greenbriar, Houston, TX 77005, USA. Fax +1 7137987940.

Abstract and commentary also published in ACP Journal Club.

Positive and negative likelihood ratios for 3 screening tests for alcohol use disorders (diagnostic standard DSM-IV)

\begin{tabular}{|c|c|c|c|c|c|c|}
\hline \multirow[b]{2}{*}{ Patient group } & \multicolumn{3}{|c|}{ Positive likelihood ratios* $(95 \%$ CI) } & \multicolumn{3}{|c|}{ Negative likelihood ratios* $(95 \%$ CI) } \\
\hline & $C A G E$ & $S A A S T$ & $A U D I T$ & $C A G E$ & SAAST & $A U D I T$ \\
\hline White men & $3.0(2.0$ to 4.4$)$ & $2.8(1.9$ to 4.1$)$ & $3.6(2.6$ to 4.8$)$ & $0.4(0.2$ to 0.7$)$ & $0.4(0.2$ to 0.7$)$ & $0.1(0.03$ to 0.3$)$ \\
\hline White women & $4.8(2.8$ to 7.7$)$ & $2.4(1.2$ to 4.5$)$ & $10.3(6.4$ to 16.4$)$ & $0.6(0.4$ to 0.9$)$ & $0.8(0.7$ to 1.0$)$ & $0.3(0.2$ to 0.5$)$ \\
\hline African-American men & $1.9(1.0$ to 3.2$)$ & $1.9(1.2$ to 2.9$)$ & $5.5(3.3$ to 9.1$)$ & $0.7(0.4$ to 1.0$)$ & $0.6(0.3$ to 0.9$)$ & $0.2(0.1$ to 0.5$)$ \\
\hline African-American women & $9.4(5.5$ to 15.2$)$ & $12.3(6.8$ to 21.7$)$ & $13.5(8.2$ to 21.9$)$ & $0.4(0.2$ to 0.6$)$ & $0.4(0.3$ to 0.6$)$ & $0.2(0.1$ to 0.4$)$ \\
\hline Mexican-American men & $3.4(1.6$ to 7.0$)$ & $2.9(1.6$ to 5.3$)$ & $3.4(2.3$ to 5.1$)$ & $0.7(0.4$ to 0.9$)$ & $0.6(0.4$ to 0.8$)$ & $0.1(0.03$ to 0.4$)$ \\
\hline Mexican-American women & 4.7 (1.7 to 11.6$)$ & $1.8(0.6$ to 4.9$)$ & $6.0(3.9$ to 9.0$)$ & $0.8(0.6$ to 1.0$)$ & $0.9(0.7$ to 1.0$)$ & $0.3(0.1$ to 0.5$)$ \\
\hline
\end{tabular}

*See definition in glossary.

\section{Commentary}

Steinbauer et al provide a convincing study supporting the superiority of the AUDIT over the CAGE and the SAAST in detecting alcohol problems among white, African-American, and MexicanAmerican primary care patients. The AUDIT has other advantages: it is brief; it can be used as a pencil-paper waiting room screen; both "at risk" alcohol users and alcohol dependent users are identified; questions address issues of consumption and harmful use; and it assesses current and lifetime diagnostic timeframes.

This study appears carefully planned and implemented and has relatively few methodological flaws. The results will help clinicians working with white, African-American, or Mexican-American patients in most primary care settings. A broader challenge this study does not address, however, is the diagnosis of occult alcohol problems. Many patients with alcohol use disorders minimise or deny consumption or related problems to avoid adverse social consequences. All 3 screening tests and the diagnostic standard used in this study rely heavily on the integrity of patients' self reports. They may well offer reproducible or comparable (ie, reliable) results. Their validity, however, may be differentially diminished in ways this study (and most others) would not detect when used for patients with occult alcohol problems. Reduced validity may occur in occupational medicine settings (eg, military medical settings) wherein open and honest disclosure may adversely affect the respondent. To im- prove detection of occult alcohol problems, a diagnostic standard should use multiple detection methods such as patient reporting, biochemical markers, longitudinal assessment, and corroborating historians. Such studies are seldom done, however, as they are expensive, difficult, and present ethical dilemmas. Without such studies, clinicians can place greater faith in the value of these self report measures when they yield positive results. Negative results must be viewed with scepticism in the context of all available clinical information.

Rena Ferguson, MD, MPH

Charles C Engel Jr, MD, MPH

Uniformed Services University Washington, DC, USA 